\title{
Safe and Sample-Efficient Reinforcement Learning Algorithms for Factored Environments
}

\author{
Thiago D. Simão \\ Delft University of Technology, The Netherlands \\ t.diassimao@tudelft.nl
}

\begin{abstract}
Reinforcement Learning (RL) deals with problems that can be modeled as a Markov Decision Process (MDP) where the transition function is unknown. In situations where an arbitrary policy $\pi$ is already in execution and the experiences with the environment were recorded in a batch $\mathcal{D}$, an RL algorithm can use $\mathcal{D}$ to compute a new policy $\pi^{\prime}$. However, the policy computed by traditional RL algorithms might have worse performance compared to $\pi$. Our goal is to develop safe RL algorithms, where the agent has a high confidence that the performance of $\pi^{\prime}$ is better than the performance of $\pi$ given $\mathcal{D}$. To develop sample-efficient and safe RL algorithms we combine ideas from exploration strategies in RL with a safe policy improvement method.
\end{abstract}

\section{Model-based Exploration in RL}

To find the optimal policy quickly, the R-max algorithm [Brafman and Tennenholtz, 2002] incentivizes the agent to explore unknown parts of the environment in early stages of the learning process. To do so, it keeps track of a set of stateaction pairs considered known:

$$
K_{m}=\{(s, a) \in S \times A \mid n(s, a) \geq m\},
$$

where $n(s, a)$ is the number of times the agent has applied action $a$ in state $s$, and $m$ is a threshold to consider a stateaction pair known.

Often, the state space $S$ can be represented by a set of state factors $X=\left\{X_{1}, \cdots, X_{|X|}\right\}$ where each factor has a domain $\operatorname{Dom}\left(X_{i}\right)$. When these factors are highly independent, a Factored MDP (FMDP) can compactly represent an MDP, using a dependence function $D: S \times A \times X \rightarrow \mathcal{I}$ that indicates the commonalities among different factors, where $\mathcal{I}$ is a set of dependency identifiers [Strehl, 2007]. The probabilistic transition function can be compactly represented:

$$
T\left(s^{\prime} \mid s, a\right)=\prod_{i=1}^{|X|} P\left(s_{i}^{\prime} \mid D\left(s, a, X_{i}\right)\right),
$$

where $s_{i}^{\prime}$ is the value of $X_{i}$ in the next state $s^{\prime}$.

The factored R-max algorithm is a direct extension of Rmax for FMDPs [Guestrin et al., 2003]. It maintains an estimate of each transition component distribution and decides which state-action pairs are known or not according to these estimates. This algorithm only considers as known parts of the environment where the estimate of all transition components have been experienced enough times. In particular, given a minimum number of samples for each factor $\vec{m}=\left\langle m_{1}, \ldots, m_{|X|}\right\rangle$ and the counters of each transition component $n(j)$, the set of known state-action pairs is constructed as follows:

$$
K_{\vec{m}}=\left\{(s, a) \in S \times A \mid \forall X_{i}: n\left(D\left(s, a, X_{i}\right)\right) \geq m_{i}\right\} .
$$

\section{Safe Policy Improvement}

Safe Policy Improvement (SPI) addresses the question of how to compute a new policy $\pi$ that outperforms the behavior policy $\pi_{b}$ with high confidence $1-\delta$, given a batch of previous interactions $\mathcal{D}$ and an admissible error $\zeta$.

The SPI by Baseline Bootstrapping (SPIBB) framework is a model-based approach that guarantees safety by bootstrapping unknown parts of the approximated model with the behavior policy $\pi_{b}$ [Laroche et al., 2019]. Formally, the set of bootstrapped state-action pairs $\mathfrak{B}_{m}$ is the complement of the set $K_{m}$ (1). This way, the SPIBB algorithms guarantee to perform at least as well as the behavior policy and do not rely on a safety test, in contrast to other SPI algorithms.

The policy-based $\Pi_{b}$-SPIBB algorithm attributes the same probability to bootstrapped pairs as the behavior policy, which restricts the policy space to

$$
\Pi_{b}=\left\{\pi \mid \pi(s, a)=\pi_{b}(s, a): \forall \pi \in \Pi, \forall(s, a) \in \mathfrak{B}_{m}\right\} .
$$

Laroche et al. [2019] prove that if $m=\frac{2}{\epsilon^{2}} \log \frac{|S||A| 2^{|S|}}{\delta}$ then the $\Pi_{b}$-SPIBB algorithm is safe, where $\epsilon$ is a bound on the $L_{1}$ distance between the estimated transition function and the true transition function, that depends on the precision parameter $\zeta$.

The $\Pi_{b}$-SPIBB algorithm can change the policy if a subset of the state-action pairs is well known, therefore it ca be less conservative than other SPI algorithms. Nevertheless, when the problem is described by a set of factors, $m$ grows exponentially in the number of factors. In the next section we show that, by taking in account the independence between features, it is possible to exploit the factored representation of the problem using a minimum number of samples that is only polynomial in the number of parameters of the FMDP. 


\section{Main Contributions}

To exploit the structure of an FMDP we proposed the Factored $\Pi_{b}$-SPIBB algorithm, an adaptation of $\Pi_{b}$-SPIBB algorithm for factored environments [Simão and Spaan, 2019]. This algorithm takes an extra input: the dependency function $D$ that determines which transition components must be estimated.

First, the algorithm estimates each transition component according to $\mathcal{D}$ using the same counters as the factored $\mathrm{R}$ max algorithm. Second, it redefines the set of state-action pairs to be bootstrapped $\mathfrak{B}_{\vec{m}}$ as the complement of the set of known state-action pairs $K_{\vec{m}}$ (2). In this way, given $\mathfrak{B}_{\vec{m}}$ and the behavior policy $\pi_{b}$, the constrained policy space $\Pi_{b}$ can be computed using (3). Finally, the algorithm searches for an optimal policy in $\Pi_{b}$, however, in this case the transition function $\hat{T}(\cdot \mid s, a)$ is estimated according to the estimate of each transition component.

The theoretical analysis shows that it is possible to bound the probability that the Factored $\Pi_{b}$-SPIBB algorithm computes a policy worse than the behavior policy. To do so it is necessary to define $\vec{m}$ such that

$$
m_{i}=\frac{2|X|^{2}}{\epsilon^{2}} \log \frac{|\mathcal{Q}| 2^{\left|\operatorname{Dom}\left(X_{i}\right)\right|}}{\delta}, \forall i \in[1,|X|] .
$$

Therefore, given a desired $\epsilon$, the term $|A||S|$ is replaced by $|\mathcal{Q}|$ and $|S|$ is reduced to $\left|\operatorname{Dom}\left(X_{i}\right)\right|$. This comes at the lower cost of adding a term polynomial in the number of variables $|X|^{2}$ (necessary to bound the error of each component distribution). In domains where the features are highly independent from each other these results reduce significantly the number of samples necessary to improve the behavior policy.

Next, we relax the assumption that the dependency function $D$ is known a priori, by proposing the Structure Learning $\Pi_{b}$-SPIBB that integrates different structure learning algorithms [Diuk et al., 2009; Strehl et al., 2007] in the SPIBB framework.

The empirical analysis shows a large difference in the number of samples necessary to change the behavior policy of flat SPI algorithms and their factored counterparts. In the SysAdmin domain (Figure 1), the Factored $\Pi_{b}$-SPIBB algorithm manages to compute policies better than the behavior policy given batches with only 100 trajectories, in contrast to the $\Pi_{b}$-SPIBB algorithm, that only shows improvement when $|\mathcal{D}| \geq 2000$. Finally, we find that even without the prior knowledge about the dependency function $D$, the Factored $\Pi_{b}$-SPIBB algorithm equipped with the structure learning kmeteorologists algorithm can also exploit the independence between features, finding improved policies with 1000 trajectories.

\section{Future Work}

Some directions for future work include: extension of other model-based safe RL methods to factored environments, such as the robust approach by Petrik et al. [2016]; exploitation of other types of structure in the SPIBB framework such as decision trees and relation between objects; and development of a general SPIBB framework to be applied under different assumptions of prior knowledge.

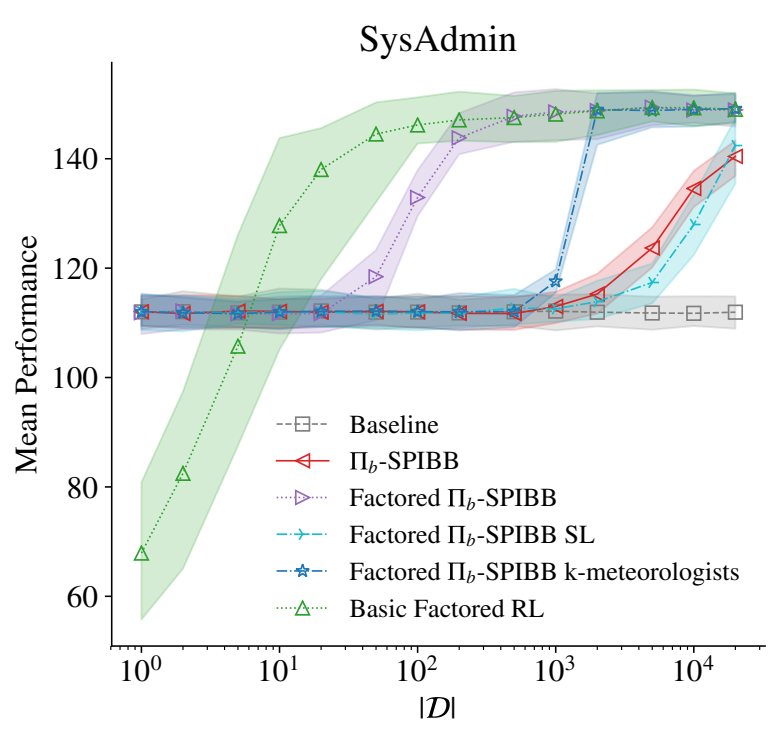

Figure 1: Average performance of the policy computed by different algorithms ( $y$-axis). The $x$-axis shows the number of trials in $\mathcal{D}$.

\section{Acknowledgments}

This research received funding from the Netherlands Organisation for Scientific Research (NWO).

\section{References}

[Brafman and Tennenholtz, 2002] Ronen I. Brafman and Moshe Tennenholtz. R-MAX - A General Polynomial Time Algorithm for Near-Optimal Reinforcement Learning. JMLR, 3:213-231, 2002.

[Diuk et al., 2009] Carlos Diuk, Lihong Li, and Bethany R. Leffler. The Adaptive $k$-meteorologists Problem and Its Application to Structure Learning and Feature Selection in Reinforcement Learning. In ICML, pages 249-256. ACM, 2009.

[Guestrin et al., 2003] Carlos Guestrin, Daphne Koller, Ronald Parr, and Shobha Venkataraman. Efficient Solution Algorithms for Factored MDPs. JAIR, 19:399-468, 2003.

[Laroche et al., 2019] Romain Laroche, Paul Trichelair, and Rémi Tachet des Combes. Safe policy improvement with baseline bootstrapping. In ICML, pages 3652-3661. PMLR, 2019.

[Petrik et al., 2016] Marek Petrik, Mohammad Ghavamzadeh, and Yinlam Chow. Safe Policy Improvement by Minimizing Robust Baseline Regret. In NeurIPS, pages 2298-2306. Curran Associates, Inc., 2016.

[Simão and Spaan, 2019] Thiago D. Simão and Matthijs T. J. Spaan. Safe Policy Improvement with Baseline Bootstrapping in Factored Environments. In AAAI. AAAI Press, 2019.

[Strehl et al., 2007] Alexander L. Strehl, Carlos Diuk, and Michael L. Littman. Efficient Structure Learning in FactoredState MDPs. In AAAI, pages 645-650. AAAI Press, 2007.

[Strehl, 2007] Alexander L Strehl. Model-Based Reinforcement Learning in Factored-State MDPs. In 2007 IEEE International Symposium on Approximate Dynamic Programming and Reinforcement Learning, pages 103-110. IEEE, 2007. 